\title{
Professor Xiaoyan Wang's Experience in Treating Tinnitus with Qi Vacuity Pattern by Using Qi Monism
}

\author{
Linlin Yang1, 2, Yihan Liu1, Xiaoyan Wang2* \\ ${ }^{1}$ Shaanxi University of Chinese Medicine, Xianyang 712046, Shaanxi Province, China \\ ${ }^{2}$ Xi'an Hospital of Traditional Chinese Medicine, Xi'an 710021, Shaanxi Province, China \\ *Corresponding author: Xiaoyan Wang, wxiaoyan3046@163.com
}

\begin{abstract}
Tinnitus refers to the abnormal sound hallucinations in the ear or inside the skull when the patient lacks outside sound. In recent years, the incidence of tinnitus is increasing gradually, which seriously affects the quality of life of patients. Reducing tinnitus symptoms is one of the main purposes of clinical treatment. Professor Xiaoyan Wang applied the monism of Qi, combined with the dialectics of the six channels and the therapeutic effect was remarkable.
\end{abstract}

Keywords: Monism of Qi; Xiaoyan Wang; Tinnitus of Qi vacuity pattern

Publication date: September 2021; Online publication: September 30, 2021

\section{Introduction}

Tinnitus is a subjective symptom that occurs alone or together with other diseases or symptoms. Clinically, it is often accompanied by hearing loss, headache, dizziness, sleep disorder, inattention, emotional anxiety and depression ${ }^{[1]}$. Traditional Chinese medicine has rich experience and unique advantages in the treatment of tinnitus. Professor Xiaoyan Wang, chief physician, is a famous traditional Chinese medicine in Shaanxi Province. He has been engaged in clinical practice for more than 30 years and is good at treating various brain diseases and other internal injuries and miscellaneous diseases. The authors are very honored to learn from Professor Xiaoyan Wang and attend the clinic with the teacher. Now they summarize the experience of their mentor in the treatment of tinnitus with Qi vacuity pattern as follows.

\section{Monism of Qi}

Monism of Qi holds that six Qi is the realization of one Qi, and congenital kidney Qi and acquired Stomach Qi are the root of each other. One Qi is the original Qi, original Qi is the source of life, original Qi contains people's spirit that comes from being born. $\mathrm{Ke} \mathrm{Li}$, an old Chinese medicine, summarized it as "Original Qi is a little true Yang in the Kan, is the foundation of man's life." The mystery of life lies in this, and this is congenital kidney Qi. The middle Qi is also generated by the original Qi. The middle Qi is like an axis and the limbs are like a wheel ${ }^{[2]}$. Ke Li, an old Chinese doctor, believes that no earth can make a world, and earth can produce all things. The middle earth of the human body is the spleen and stomach (middle Qi). It rises on the left and falls on the right ${ }^{[3]}$. Mediation runs continuously, and the five internal organs can be raised and live forever. Therefore, it means "earth subdues fire." The "monism of Qi" holds that the Qi is the physiological process of the body to produce the qi of the five internal organs through the rise and fall of the functioning of Qi. Ascending liver Qi is on the left, descending lung Qi is on the right, floating heart Qi is on the top, sinking kidney Qi is on the bottom, and spleen and stomach are the middle Qi which are 
in the middle. The spleen rises and the stomach falls, which is the hub of Qi movement. Therefore, the Qi of the five internal organs is actually one Qi, which is the specific embodiment of different stages of Qi movement ${ }^{[4]}$. The root of human vitality is based on the kidney and sprouts in the liver. Since things have taken root, sprouting can reflect the vitality of things. Therefore, whether the sprouting power of the liver is strong or not is another important key to human vitality. The kidney water generates, liver govern wood and grows upward. The liver qi rises left, the lung gold falls right, the functioning of Qi collects and falls, the cool and moist soil comes down, the spleen and stomach is in the middle, the supreme Yin and wet soil rises left, the Yang brightness dry soil falls right, the middle soil is the axis, the remaining viscera is the wheel, one Qi flows, circulates, and the cycle is endless ${ }^{[5]}$.

\section{Understanding tinnitus of Qi vacuity pattern from monism of Qi}

Professor Wang believes that tinnitus due to qi deficiency is mostly caused by deficiency of original Qi, and deficiency of spleen and kidney due to improper diet and emotional exhaustion. Congenital deficiency of vital energy and Yang, weakening of viscera function, dysfunction of Qi and abnormal entry and exit of Qi function will not rise at the sight of the left road first: The spleen soil are in disorder, cold, damp, phlegm and blood stasis are hidden in the soil, which will turn into heat and fire for a long time, burn the wet soil, and cause no rise on the left road ${ }^{[6]}$. Second, see the right but not to drop: If the spleen is not upbearing the clear, the stomach is not descending the turbid, and the Yang brightness is dry and hot, and will consume Qi, blood and body fluid. If the spleen cannot carry out its body fluid for the stomach and disperse essence in the lung, the lung Qi will not fall; Yang brightness does not fall, carrying turbid pathogens such as wind, fire, phlegm and blood stasis to disturb the clear orifices ${ }^{[7]}$. Third, see the A gallbladder but not to drop: The gallbladder wood generates phase fire. Qin'an Zheng thought: "Phase fire is true fire". He believed that the deeper the phase fire falls, the stronger and sufficient the Yang root, the liver Qi did not rise, the Qi deficiency and Qi sank at the bottom, then generated cold, dampness, phlegm, turbidity and blood stasis, the A gallbladder did not fall, went straight up with it, beclouded clear orifices, the functioning of Qi was chaotic, the clarity and turbidity were reversed, the turbid evil stole the Yang position, clear orifices blocked, and made a sound, which was called tinnitus.

\section{Characteristics of therapeutic drugs}

Center-Supplementing Qi-Boosting Decoction was selected for treatment. Center-Supplementing QiBoosting Decoction: Supple the center and boost Qi, strengthen the spleen, upbear the clear and promote the liver qi. Prescription is: raw Radix Astragali seu Hedysari, radix ginseng, fried Rhizoma Atractylodis Macrocephalae, Radix Angelicae sinensis, Radix Bupleuri, Rhizoma Cimicifugae, dried orangepeel, and roasted Radix glycyrrizae. The prescription takes a large amount of raw Radix Astragali seu Hedysari as the principal drug. A large amount of raw Radix Astragali seu Hedysari can strengthen the Middle Earth, fix the central axis, replenish the middle Qi and raise the liver Qi. Roasted Radix Glycyrrhizae harmonizes the internal organs, penetrates Yin and Yang, governs the positive Qi of Yin and Yang in the internal organs. Radix Ginseng replenishes the five internal organs, replenishes the true Qi of heart, kidney, lung and liver, calms the mind and benefits intelligence, and complements the body vitality of monarchs and officials. Fried Rhizoma Atractylodis Macrocephalae can help the earth Qi run, dry and moist, warm and harmonious. It is the biochemical source of Qi and blood, and can jack up the cemented medium earth. Radix Angelicae Sinensis nourishes the blood, nourishes the middle energizer to nourish the blood, replenishes the liver body, and uses Yin and Eang to help the Qi of B wood rise. Dried orangepeel dredges the collateral of the spleen and stomach, so that the medicine of the whole prescription supple but not stagnate. Radix Bupleuri, Rhizoma Cimicifugae, rise clear and reduce turbidity, and combine with Astragalus membranaceus, ginseng, Rhizoma Atractylodis Macrocephalae and radix glycyrrhizae, make the middle Qi down and drug 
upward ${ }^{[8]}$. Radix Bupleuri can also disperse stagnated liver Qi to relieve depression, harmonizing liver Qi. Rhizoma Cimicifugae disperses Yang brightness and stomach muggy ${ }^{[9]}$. Add Radix Puerariae, tasting sweet and entering into the spleen, nature flavor is spicy and scattered. The first helps the spleen Yang rise clear, Yang rises and then Yin goes up. The second helps produce saliva and slake thirst, body fluid produces when Qi goes up, so it restricts the large dose of astragalus tepid. Take an overall view of the whole prescription, cultivate and supplement the Central Qi, strengthen the central axis of movement, rise and fall phase causes, and clear and turbid reset. Add and subtract are as follows: If the liver Qi is depressed and turns into fire due to depression, irritability, upset and bitter mouth, consider adding Radix Curcumae, Radix Aucklandiae, burnt Fructus Gardeniae, wine Radix Scutellariae, etc. If the patient is thirsty, add Radix Trichosanthis. If the patients are constipation, add raw Gypsum Fibrosum and wine-treated rhubarb.

\section{Summary}

Professor Wang believes that the majority of patients with tinnitus syndrome of Qi vacuity pattern are middle-aged and old, which are induced or aggravated by fatigue and emotion, and can be combined with psychological comfort therapy when necessary. This article is the authors' summary of their mentor's clinical treatment experience, lack of evidence-based medicine data, and there are some insufficiencies, which need to be further studied and improved.

\section{Disclosure statement}

The author declares no conflict of interest.

\section{References}

[1] Chen Y, Han M, Wang M, et al., 2019, Progress in the Diagnosis and Treatment of Tinnitus. Clinical Misdiagnosis and Mistreatment, 32(1): 109-113.

[2] Wu LZ, 2015, Study on the Association between Ke Li 's thought on Medicine and Treatise on Febrile Diseases. Jiangsu Province: Nanjing University of Chinese Medicine.

[3] Zhou NY, et al., 2015, Based on the Monism of Qi, Discussion on the Treatment of Children Tumor from the Restoration of "Yang Brightness and Machine Descending." Guangming Journal of Chinese Medicine, 30 (10).

[4] Wen LT, et al., 2017, Ying Lv's Clinical Experience in the Treatment of Children's Tumors Based on the Theory of "Yang Brightness's Great Descending Machine." Liaoning Journal of Traditional Chinese Medicine. 44 (11).

[5] Gao J, et al., 2019, Clinical Experience in Treating Tumour. China Journal of Traditional Chinese Medicine and Pharmacy, 34(10).

[6] Wen Z, 2020, Wang Xiaoyan's Experience in Treating Myasthenia Gravis with Qi Monism. Guide of Traditional Chinese Medicine. 26 (11).

[7] Luo M, 2020, Summary of Professor Wang Xiaoyan's Experience in Treating Internal Injuries and Miscellaneous Diseases by Time. Tianjin Traditional Chinese Medicine. 37 (05).

[8] Ying X, Song F, 2012, Analysis of the Academic thought of "Spleen and Stomach Theory." Modern Distance Education of Chinese Medicine, 10 (19): 3-4.

[9] Li D, 2005, "On the Spleen and Stomach: On the Victory and Decline of the Spleen and Stomach." Beijing: People's Medical Publishing House, 7-16. 